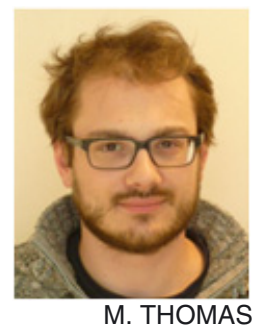

\title{
Approche du LNE pour la réalisation et la mise en pratique du kilogramme dans sa nouvelle définition
}

\section{Approach of the LNE to the realization and the mise en pratique of the kilogram in its new definition}

\author{
Matthieu THOMAS ${ }^{1}$, Patrick ESPEL ${ }^{1}$, Djamel ZIANE ${ }^{1}$, François PIQUEMAL ${ }^{1}$, Patrick PINOT ${ }^{2}$, \\ Patrick JUNCAR ${ }^{2}$, Zaccaria SILVESTRI ${ }^{2}$, Mark PLIMMER ${ }^{2}$, Franck PEREIRA DOS SANTOS ${ }^{3}$, \\ Sébastien MERLET ${ }^{3}$, Florian BEAUDOUX ${ }^{1}$, Djilali BENTOUATI ${ }^{1}$, Frédéric BRUNET ${ }^{1}$, \\ Patrick JEANJACQUOT ${ }^{1}$ et Tanguy MADEC ${ }^{1}$

\footnotetext{
1 LNE (Laboratoire national de métrologie et d'essais), 29 avenue Roger Hennequin, 78197 Trappes Cedex, France, matthieu.thomas@lne.fr.

${ }^{2}$ LNE-LCM (laboratoire commun de métrologie LNE - Cnam), Cnam (Conservatoire national des arts et métiers), 61 rue du Landy, 93210 La Plaine St-Denis, France.

3 LNE-SYRTE, Observatoire de Paris, PSL Research University, CNRS, Sorbonne Universités, UPMC Paris 06, France.
}

\section{Résumé}

Le kilogramme est la dernière unité du Système international d'unités (SI) encore définie par un artefact : le prototype international du kilogramme (International Prototype of the Kilogram ou IPK). En 100 ans, des comparaisons entre l'IPK, ses copies officielles et les prototypes nationaux ont montré une variation relative de masse de l'ordre de $5 \times 10^{-8}$. Après un bref rappel sur l'unité de masse, cet article expose la nécessité de redéfinir le kilogramme. Il explique le choix de la constante de Planck $h$ comme base d'une définition telle qu'envisagée par la CGPM en 2018. L'article présente les derniers résultats obtenus avec les balances du watt qui permettent aujourd'hui d'établir un lien entre $h$ et une masse macroscopique avec des incertitudes relatives de quelques $10^{-8}$. Enfin, l'article présente la position adoptée en France par le laboratoire national de métrologie pour la «mise en pratique » du kilogramme et sa dissémination après sa redéfinition en 2018.

MOTS CLÉS : UNITÉ DE MASSE, KILOGRAMME, REDÉFINITION DU KILOGRAMME, CONSTANTE DE PLANCK, BALANCE DU WATT.

\footnotetext{
Abstract

The kilogram is the last unit of the International System of Units (SI) still defined by an artefact, namely the International Prototype of the Kilogram (IPK). Comparisons carried out over 100 years between $I P K$, its official copies and national prototypes have revealed a mass
}

change (in relative term) of five parts in $10^{8}$. After a brief history of the unit of mass, this paper underlines the need to redefine the kilogram with reference to a fixed numerical value of a fundamental constant. It explains why the Planck constant $h$ was chosen and gives the last results obtained with watt balance experiments nowadays able to link $h$ to a macroscopic mass to within a few parts in $10^{8}$. Finally, it proposes a possible route for the national metrology institute for the mise en pratique of the kilogram after its redefinition.

KEY WORDS: UNIT OF MASS, KILOGRAM, REDEFINITION OF THE KILOGRAM, PLANCK CONSTANT, WATT BALANCE.

Dans cet article, les auteurs retracent brièvement l'histoire du kilogramme et rappellent sa définition actuelle. Les problématiques liées à cette définition sont ensuite décrites : elles pourraient être résolues en raccordant l'unité de masse à une ou plusieurs constantes physiques fondamentales. Le choix de la valeur de la constante de Planck $h$ pour redéfinir le kilogramme est discuté et les derniers résultats obtenus à partir des expériences «balance du watt » et «sphère de silicium » sont donnés. Enfin, la stratégie de «mise en pratique » du kilogramme qui sera adoptée par le laboratoire, suite à l'adoption d'une nouvelle définition en 2018, est exposée. 


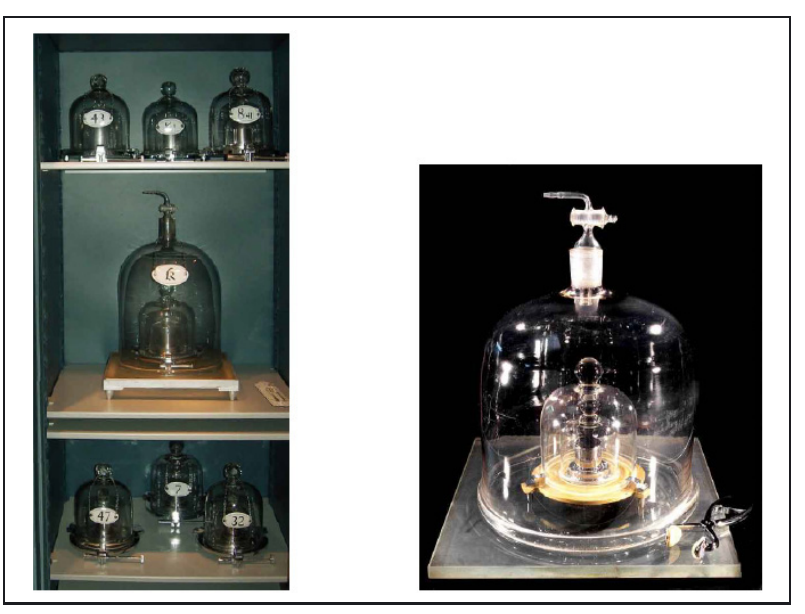

Fig. 1. - Figure de gauche (d'après Bordé [1]) : Coffre contenant le prototype international du kilogramme, conservé dans l'air au BIPM sous trois cloches de verre ainsi que ses six copies officielles; figure de droite (d'après BIPM [2]) : Copie du prototype international du kilogramme ou IPK.

\section{Définition actuelle du kilogramme}

En France, la Convention nationale en 1793 prévoit la création d'étalons pour la longueur et la masse. La détermination de l'unité de masse a nécessité deux campagnes. La première, en 1792-1793, a permis de définir l'unité provisoire, le grave (du latin gravis, lourd, pesant), la seconde, en 1798-1799, l'unité définitive, le kilogramme. Le kilogramme est alors défini par un artefact cylindrique en platine, connu sous le nom de « Kilogramme des Archives » correspondant à la masse d'un décimètre cube d'eau à son maximum de densité (soit à environ $4{ }^{\circ} \mathrm{C}$ ).

En 1878, des copies du Kilogramme des Archives furent réalisées par la société anglaise Johnson Matthey. Parmi ces copies, fut choisi, le 28 septembre 1889, le prototype international du kilogramme ou IPK, conservant au kilogramme une magnitude proche de la précédente : il s'agit d'un cylindre de platine iridié, alliage de $90 \%$ de platine et $10 \%$ d'iridium. Son diamètre de $39 \mathrm{~mm}$ est égal à sa hauteur.

Le prototype international du kilogramme a été déposé en 1889 dans un caveau (à 9 mètres sous terre) du pavillon de Breteuil au Bureau international des poids et mesures (BIPM) à Sèvres (France). Il est placé sous trois cloches de verre dans l'air (fig. 1) [1,2] et enfermé dans une armoire forte commandée par trois clefs. La première est conservée par le directeur des Archives de France, la deuxième par le directeur du BIPM, la troisième par le président du Comité international des poids et mesures (CIPM). La présence simultanée des trois clefs est nécessaire pour pouvoir accéder aux étalons qui ne sont visibles qu'une seule fois par an.

Pour faire cesser l'ambiguïté qui existait dans l'usage courant sur le terme "poids », la troisième Conférence générale des poids et mesures (CGPM) en 1901 confirma que « le kilogramme est l'unité de masse; il est égal à la masse du prototype international du kilogramme [3]. » Il en résulte que la masse du prototype international du kilogramme, symbolisé par la lettre gothique $\mathfrak{K}$ [4], est par définition toujours égale à $1 \mathrm{~kg}$ exactement, même si la quantité de matière que contient l'artefact évolue.

À ce jour, le kilogramme est la dernière des sept unités de base [5] du Système international d'unités (SI) qui soit toujours directement définie par un objet matériel plutôt que par une propriété physique fondamentale permettant de réaliser l'unité de base dans les laboratoires nationaux.

\section{Problématiques liées à la définition actuelle du kilogramme}

La définition et la réalisation du kilogramme sont confondues dans l'artefact $\mathfrak{K}$. Cette confusion renvoie à plusieurs problèmes :

- La définition même n'est universelle ni dans l'espace, ni dans le temps, ni en amplitude : il est nécessaire de disposer de l'IPK pour réaliser l'unité de masse dont la valeur a été fixée à $1 \mathrm{~kg}$ exactement et l'IPK ne peut être remplacé par un autre prototype (en cas de détérioration ou de destruction).

- La maîtrise des conditions de conservation du prototype international du kilogramme est nécessaire pour contrôler sa stabilité à long terme. Ceci implique de limiter au strict nécessaire l'utilisation du prototype mais aussi de lui appliquer, uniquement à l'occasion d'une campagne de vérification des prototypes nationaux, une méthode de nettoyagelavage «type BIPM» (méthode utilisant des solvants tels que l'éther et l'éthanol mais aussi de la vapeur d'eau $[6,7]$ ) pour éliminer autant que possible les inévitables contaminations de la surface du prototype international du kilogramme (ainsi que des autres prototypes et étalons de référence en platine iridié) [8].

- L'unicité de référence (sa disponibilité en un seul lieu) rend la vérification des quelques soixante prototypes nationaux possible seulement s'ils sont tous rassemblés au BIPM pour une durée de deux ou trois années. Ces vérifications « ordinaires » n’ont donc lieu que tous les cinquante ans environ depuis l'adoption de l'IPK pour réaliser l'unité de masse : distribution et certification des prototypes en 1889 [2], deuxième vérification périodique en 1946-1953 [9], troisième vérification périodique en 1988-1992 [10]. Une comparaison extraordinaire a eu lieu en 2014 en prévision de la refonte du SI [11].

Ces trois vérifications périodiques entre le prototype international du kilogramme et les six témoins conservés dans les mêmes conditions que l'IPK montrent des divergences d'évolution dans le temps (fig. 2). La dispersion de l'évolution relative de ces six étalons de masse sur un siècle est de l'ordre de $5 \times 10^{-8}$ et même de $1 \times 10^{-7}$ si l'on considère l'ensemble des étalons nationaux. Une étude de l'évolution des prototypes du kilogramme a été effectuée à l'occasion de la troisième vérification [10]. Elle a montré que la dérive moyenne des prototypes conservés 


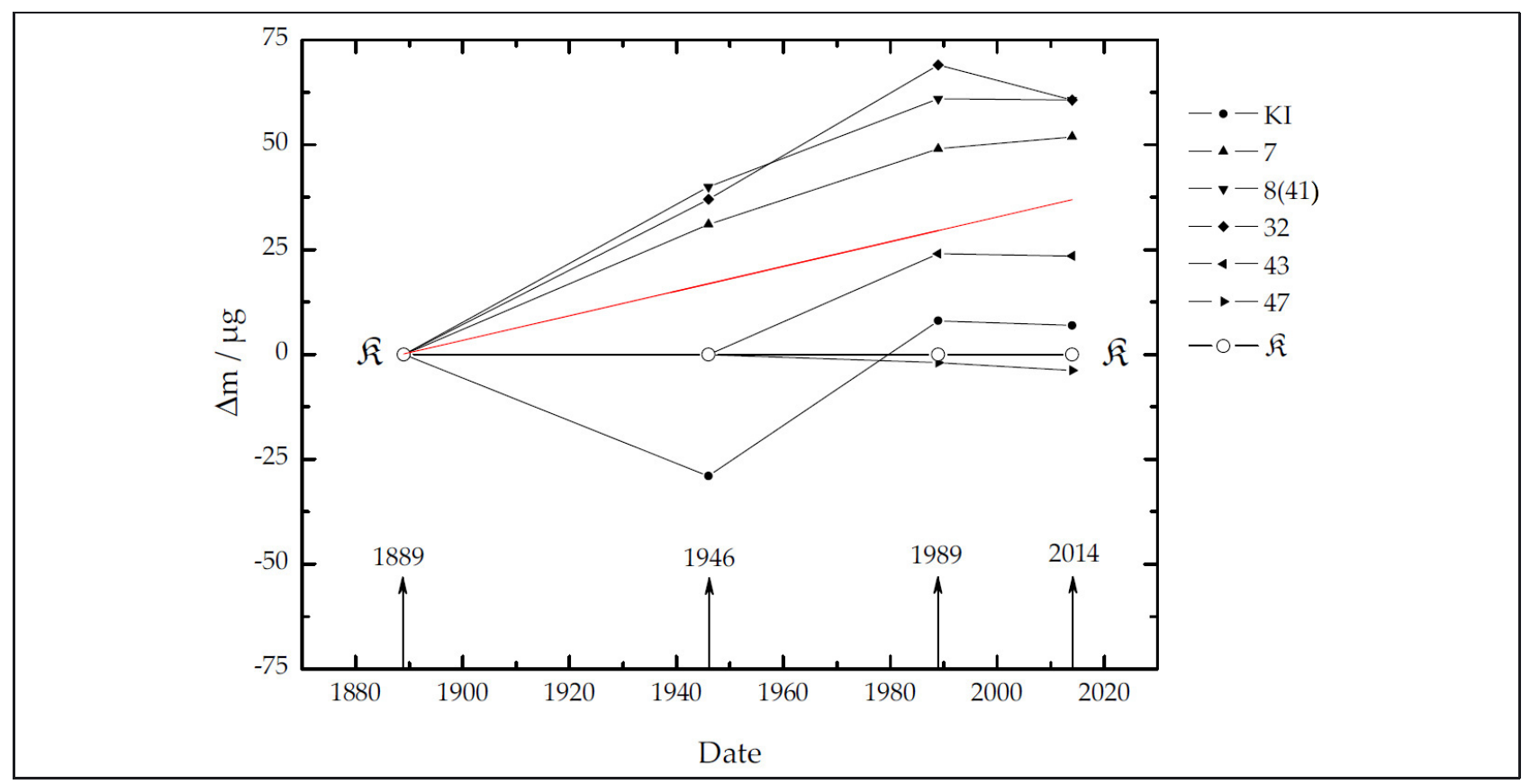

Fig. 2. - Évolutions de l'écart de masse des six témoins officiels (KI, 7, 8(41), 32, 43 et 47) à la masse du prototype international du kilogramme $(\mathfrak{K})$. La droite rouge, régression linéaire des données, présente une pente de l'ordre de $+0,25 \mu \mathrm{g}$ par an.

au BIPM dans les meilleures conditions et utilisés avec le plus grand soin était $+0,25 \mu \mathrm{g}$ par an alors qu'elle était de l'ordre de $+1 \mu \mathrm{g}$ par an en prenant en compte l'ensemble des prototypes nationaux. Ces dérives sont relatives à la masse de l'IPK supposée constante et égale exactement à un kilogramme par définition. La dérive absolue de la masse de l'IPK est actuellement inaccessible. Il a seulement été montré qu'elle est inférieure à $10^{-6}$ en valeur relative mais elle pourrait se révéler être significative par rapport à une constante fondamentale de la physique permettant de réaliser l'unité de masse à quelques $10^{-8}[12,13]$.

Le fait de voir l'écart de masse entre les six témoins et le prototype international du kilogramme évoluer est un indice fort de l'évolution de la masse de l'IPK par rapport à une référence stable, comme la masse d'une particule. Alors, à prendre la définition de l'unité de masse, on arrive à la conclusion que la masse du prototype international du kilogramme, $m(\mathfrak{K})$, ne varie pas, tandis que la masse d'un électron par exemple dans le SI, varierait au cours des décennies : cela est a minima insatisfaisant. Par ailleurs, la tendance à l'accroissement de masse des copies officielles (relativement à la masse de l'IPK) observée durant les trois premières vérifications $(1889$, 19461953, 1988-1992) n'a pas été confirmée par la dernière vérification. Ces résultats montrent que la différence de masse entre l'IPK et ses copies officielles n'a changé en moyenne que de $1 \mu \mathrm{g}$ au cours des 25 dernières années : la dérive des copies par rapport à l'IPK n'est pas prévisible.

En sus de l'arrêt de la dérive observée précédemment, l'unité de masse maintenue au BIPM depuis la troisième vérification à partir d'une dizaine de kilogrammes en platine iridié a été déterminée comme erronée de $35 \mu \mathrm{g}$ [14] relativement à la masse de l'IPK (soit
$3,5 \times 10^{-8}$ en valeur relative). L'évolution de ce décalage dans le temps (de 1992 à 2014) a été modélisée en supposant une diminution de masse des prototypes proportionnelle au nombre de pesées dans les comparateurs de masse. Les corrections sur les valeurs des étalons nationaux ont été calculées a posteriori. L'incertitude type associée a été déterminée à $3 \mu \mathrm{g}$ et l'ensemble de ces informations transmises aux laboratoires nationaux de métrologie à la fin 2014 : l'unicité de la réalisation du kilogramme rend les erreurs difficiles à détecter, puisque des inter-comparaisons entre réalisations primaires ne sont pas possibles.

Pour des raisons de disponibilité, de pérennité et d'universalité du kilogramme, il est donc indispensable d'en changer la définition, actuellement fondée sur un artefact matériel unique. Cet artefact, de masse constante par convention, est en réalité physiquement variable du fait des effets de l'usure, du dégazage et de la contamination superficielle (principalement, phénomènes de sorption et de dépôt de fines particules d'aérosol) et il est impossible à copier à l'identique, c'est-à-dire au microgramme près en termes de masse.

\section{Raccordement du kilogramme à une constante fondamentale}

\subsection{Quelle constante fondamentale choisir pour définir le kilogramme?}

Des principes physiques variés, comme le «comptage » d'ions pour former une masse d'atomes d'une dizaine de grammes [15] ou encore la lévitation d'une masse supraconductrice $[16,17]$ ont été envisagés pour établir le lien entre le kilogramme et une ou plusieurs constantes fondamentales de la physique. 
Après des réflexions sur les nouvelles définitions, et sur le choix des constantes qu'il serait le plus pertinent de fixer [18-20], et bien qu'une définition fondée sur la constante d'Avogadro $N_{\mathrm{A}}$ [21] - selon laquelle le kilogramme serait constitué d'un nombre donné d'atomes soit conceptuellement simple, un consensus s'est dégagé pour déterminer le kilogramme à partir de la valeur de la constante de Planck [22] pour les raisons suivantes :

- La constante de Planck $h$ fait partie des «trois constantes dimensionnées les plus fondamentales » de la physique [23] avec la vitesse de la lumière $c$ et la constante de gravitation $G$.

- Fixer $h$ indépendamment de $N_{\mathrm{A}}$ permet de définir la mole comme une quantité de matière, grandeur indépendante de la masse.

- Enfin, fixer $h$ et la valeur de la charge élémentaire $e$ implique [18] que la constante de Josephson $K_{\mathrm{J}}$ et la constante de von Klitzing $R_{\mathrm{K}}$ auront des valeurs exactes dans le SI, considérant la validité des expressions théoriques : $K_{\mathrm{J}}=2 e / h$ et $R_{\mathrm{K}}=h / e^{2}$ (la validité de ces deux expressions a été démontrée avec une incertitude de $2 \times 10^{-8}$ [24]).

La modification de la définition du kilogramme s'inscrirait alors dans une redéfinition globale des unités du SI, permettant d'élaborer un système d'unités reposant entièrement sur des constantes fondamentales.

\subsection{Nouvelle définition du kilogramme}

Dans un premier temps, il s'agirait d'effectuer des déterminations de la constante de Planck dans le SI actuel (et donc avec la masse du prototype international du kilogramme exactement égale à $1 \mathrm{~kg}$ ) au moyen de balances du watt ou de sphères de monocristal de silicium isotopiquement pur caractérisées par la méthode XRCD (X-ray crystal density), les expériences les plus performantes en termes d'incertitudes. Dès que la robustesse de ces différentes déterminations sera établie (cohérence et incertitude), la constante de Planck sera fixée sur la base de mesures effectuées par rapport au prototype international comme référence. Elle deviendra alors elle-même la valeur de référence à la place du prototype international permettant de déterminer la masse sous vide d'un étalon de transfert. Cet étalon dont on connaît les principales caractéristiques (coefficients de sorption, masse volumique...) pourra ensuite être comparé dans l'air au prototype international du kilogramme ou à tout autre étalon de référence. Les balances du watt ou les sphères de silicium constitueront une "réalisation primaire » de l'unité de masse, point de départ de la dissémination de l'unité de masse aux utilisateurs («mise en pratique » de la définition du kilogramme).

La 24 ${ }^{\mathrm{e}}$ CGPM [25] en 2011 propose selon ce principe une nouvelle définition à constante explicite pour le kilogramme (avec une valeur à préciser pour la constante de Planck), de même que pour les autres unités de base, et cela dans le cadre d'une refonte globale des définitions des unités de base du SI, définies également à constantes explicites. La nouvelle définition du kilogramme qui devrait être adoptée est la suivante [22] : «Le kilogramme, symbole kg, est l'unité SI de masse. Il est défini en prenant la valeur numérique fixée de la constante de Planck égale à $6,626070040 \times 10^{-34}$ lorsqu'elle est exprimée en unité $\mathrm{J} \mathrm{s}$ qui est égale $\mathrm{kg} \mathrm{m}^{2} \mathrm{~s}^{-1}$ où le mètre et la seconde sont définis en termes de $c$ et $\Delta v_{\mathrm{Cs}}$ (fréquence de la transition hyperfine de l'état fondamental de l'atome de césium). ». Dans cette définition, la valeur numérique indiquée n'est pas la valeur définitive. Elle correspond à la dernière valeur recommandée de $h$ par le CODATA [24].

Cependant, lors de sa $25^{\mathrm{e}}$ session en 2014, l'assemblée constate que "malgré les progrès effectués [dans les déterminations de $h$ ], les données disponibles ne semblent pas encore suffisamment robustes pour que la CGPM adopte le SI révisé [et donc la nouvelle définition du kilogramme] » [26]. En effet, le CIPM exige que trois expériences indépendantes (incluant des balances du watt [27] et des expériences de sphère de silicium [28]), permettent d'obtenir des valeurs cohérentes de la constante de Planck, avec des incertitudes types relatives qui ne soient pas supérieures à $5 \times 10^{-8}$ et dont l'une au moins possèderait une incertitude type relative qui ne soit pas supérieure à $2 \times 10^{-8}$ [11] .

Une telle exigence sur les niveaux d'incertitude est en effet le seul moyen de garantir la continuité historique de la valeur du kilogramme (conservation de sa magnitude lors des redéfinitions successives) et de prendre en compte les besoins communs à la métrologie industrielle et à la métrologie légale en termes d'étalonnage des instruments de pesage systématiquement réalisé en masse conventionnelle. Pour cela, il est impératif que cette nouvelle définition du kilogramme n'entraîne pas d'écart significatif sur les valeurs de masse conventionnelle des poids et masses étalons de la classe d'exactitude la plus élevée $\left(E_{1}\right)$ définie selon la recommandation R111 de l'Organisation internationale de métrologie légale (OIML) : autrement dit, il est nécessaire de pouvoir étalonner des masses de $1 \mathrm{~kg}$ en acier inoxydable avec une incertitude type relative inférieure à $8 \times 10^{-8}$ [29], cela sous-entend de maîtriser l'étalonnage de masse en platine iridié à $5 \times 10^{-8}$ ou mieux.

\subsection{Les derniers résultats}

La méthode de la balance du watt a été imaginée par Kibble au NPL (Royaume-Uni) en 1974 [30]. La première balance du watt fonctionnelle a été conçue en 1980 au NPL, suivie peu après par celle du NIST (États-Unis). Quatre générations de balance du watt ont été développées au NIST jusqu'à présent, tandis que la deuxième génération de balance du watt du NPL fut transférée au NRC (Canada) en 2010. Le METAS (Suisse) a conçu deux versions depuis 1997, tandis que le BIPM poursuit le développement de sa propre expérience initiée en 2002.

Cette expérience transverse, complexe, délicate, nécessite des temps longs de maturation et des conditions que seuls peu de laboratoires nationaux de métrologie 
sont à même de fournir : conditions environnementales maîtrisées (régulation en température et humidité, protection contre les vibrations sismiques et les perturbations électromagnétiques...), conception et réalisation d'un dispositif électromécanique sophistiqué, maîtrise simultanée de grandeurs au niveau de l'état de l'art dans différents domaines (grandeurs électriques par des comparaisons à l'effet Josephson et à l'effet Hall quantique, accélération de la pesanteur mesurée localement au moyen d'un gravimètre absolu ; détermination de vitesse, nécessité d'étalons de temps et longueur; étalons de masse au meilleur des capacités métrologiques actuelles), grandes compétences en terme de savoir et savoir-faire des opérateurs...

En France, le projet de développement d'une balance du watt a été initié en 2000 au sein de l'institut national de métrologie (BNM-INM) et du laboratoire national d'essais (BNM-LNE) (la métrologie française étant pilotée à cette époque par le Bureau national de métrologie, BNM). Après 15 ans de caractérisations et de développements, deux campagnes de mesures de la constante de Planck dans l'air ont été réalisées au cours de l'été 2014 et de l'hiver 2015-2016 :

- La première mesure, avec une masse de 500 g en Alacrite XSH (superalliage à base de cobalt) est associée à une incertitude type estimée de $3,1 \times 10^{-7}$ [31]. Les éléments contribuant le plus significativement à cette incertitude sont les mesures de tension réalisés par des voltmètres étalonnés par comparaison à une diode Zener $\left(2,4 \times 10^{-7}\right)$, le terme de puissance parasite résultant de la somme des produits des forces et des moments parasites impliqués dans la phase statique par les vitesses parasites linéaires et angulaires impliquées dans la phase dynamique $\left(8,2 \times 10^{-8}\right)$ et les incertitudes de type A $\left(8 \times 10^{-8}\right)$ estimées par la valeur de l'écart type d'Allan sur une durée de 1 mois.

- La deuxième mesure a été obtenue avec une masse de $500 \mathrm{~g}$ en platine iridié. L'incertitude type estimée sur cette valeur de $h$ est de $1,4 \times 10^{-7}$ [32]. La diminution significative de l'incertitude résulte d'une meilleure gestion de la référence de tension Zener grâce à un étalonnage horaire des voltmètres et un étalonnage hebdomadaire de la diode Zener avec une référence de tension Josephson $\left(5,7 \times 10^{-8}\right)$, de la détermination de la verticalité des faisceaux des interféromètres et des capteurs de position à mieux que $100 \mu \mathrm{rad}$ ce qui permet de diminuer le terme d'erreur de puissance du fait de la diminution des vitesses parasites mesurées $\left(5,6 \times 10^{-8}\right)$ et enfin, de la diminution de l'incertitude de type $\mathrm{A}\left(7 \times 10^{-8}\right)$ par la réalisation d'un nouveau fléau monobloc et l'amélioration des performances de son asservissement en phase statique.

Les efforts se poursuivent actuellement pour réduire les différentes composantes d'incertitudes : diminution des incertitudes sur les tensions en insérant un dispositif Josephson (réseau de jonction Josephson programmable de $1 \mathrm{~V}$ ), réduction des forces et des moments parasites qui agissent sur la bobine et le fléau en améliorant le réglage du positionnement de la bobine, réduction du bruit de mesures en phase dynamique en agissant sur la configuration de la platine de translation, suppression des incertitudes liées à la poussée d'Archimède et à la détermination de l'indice de réfraction de l'air en fonctionnant sous vide...). L'objectif est d'obtenir une incertitude type relative sur $h$ de l'ordre de $5 \times 10^{-8}$ en 2017 et de l'ordre de $2 \times 10^{-8}$ dans les années suivantes.

Les résultats des différents laboratoires sont présentés à la figure 3 .

Aujourd'hui, les données disponibles semblent suffisamment robustes pour que la prochaine CGPM de 2018 adopte la nouvelle définition du kilogramme reposant sur une valeur actualisée de la constante de Planck puisque trois expériences indépendantes (sphère de silicium : IAC 2011/2015; balances du watt : NRC 2015 et NIST 2016) répondent aux exigences du CIPM.

\section{Mise en pratique de la nouvelle définition du kilogramme}

Après la redéfinition du kilogramme prévue en 2018, la masse du prototype international du kilogramme, $m(\mathfrak{K})$, sera égale à un kilogramme avec une incertitude relative de l'ordre de $10^{-8}$ égale à celle de la valeur recommandée de $h$ par le CODATA juste avant cette redéfinition. La masse du prototype international du kilogramme devra ensuite être déterminée expérimentalement par rapport à la constante de Planck. Si cette détermination était renouvelée durant quelques dizaines d'années, il serait possible de connaître la dérive absolue de l'IPK pour cette période.

Un certain nombre d'études, de communications et publications [41-45] ont traité de la dissémination de l'unité de masse dans le futur SI.

Plusieurs voies sont possibles pour la mise en pratique et la future dissémination de l'unité de masse en France (fig. 4). La problématique de la stabilité des étalons lors du passage de l'air au vide a fait l'objet de travaux dans le cadre d'un projet européen (JRP NewKilo) [46-48]. Le laboratoire ayant l'atout de disposer d'un moyen de réalisation primaire du kilogramme (la balance du watt) et de comparateurs équipés pour les passages air-vide (résolution de $0,1 \mu \mathrm{g}$ et répétabilité de l'ordre de $0,5 \mu \mathrm{g}$ ), il pourra les mettre à profit pour bâtir sa propre chaîne de dissémination et conserver son indépendance métrologique dans le domaine des masses et grandeurs apparentées : la balance du watt servirait à étalonner sous vide, avec une incertitude cible de $5 \times 10^{-8}$, des étalons de masse aux propriétés physicochimiques adaptées à un tel emploi : platine iridié, iridium pur ou Udimet 720 (superalliage à base de nickel). Ce dernier présente, comme l'iridium, une très grande dureté, une susceptibilité magnétique de volume très faible, un comportement lors du passage air-vide équivalent à celui des étalons en platine iridié. Ces étalons seraient utilisés à leur tour pour l'étalonnage d'artefacts, de même valeur nominale de masse dans l'air, fabriqués dans le même matériau et faisant le lien entre les mesures sous vide et dans l'air. 


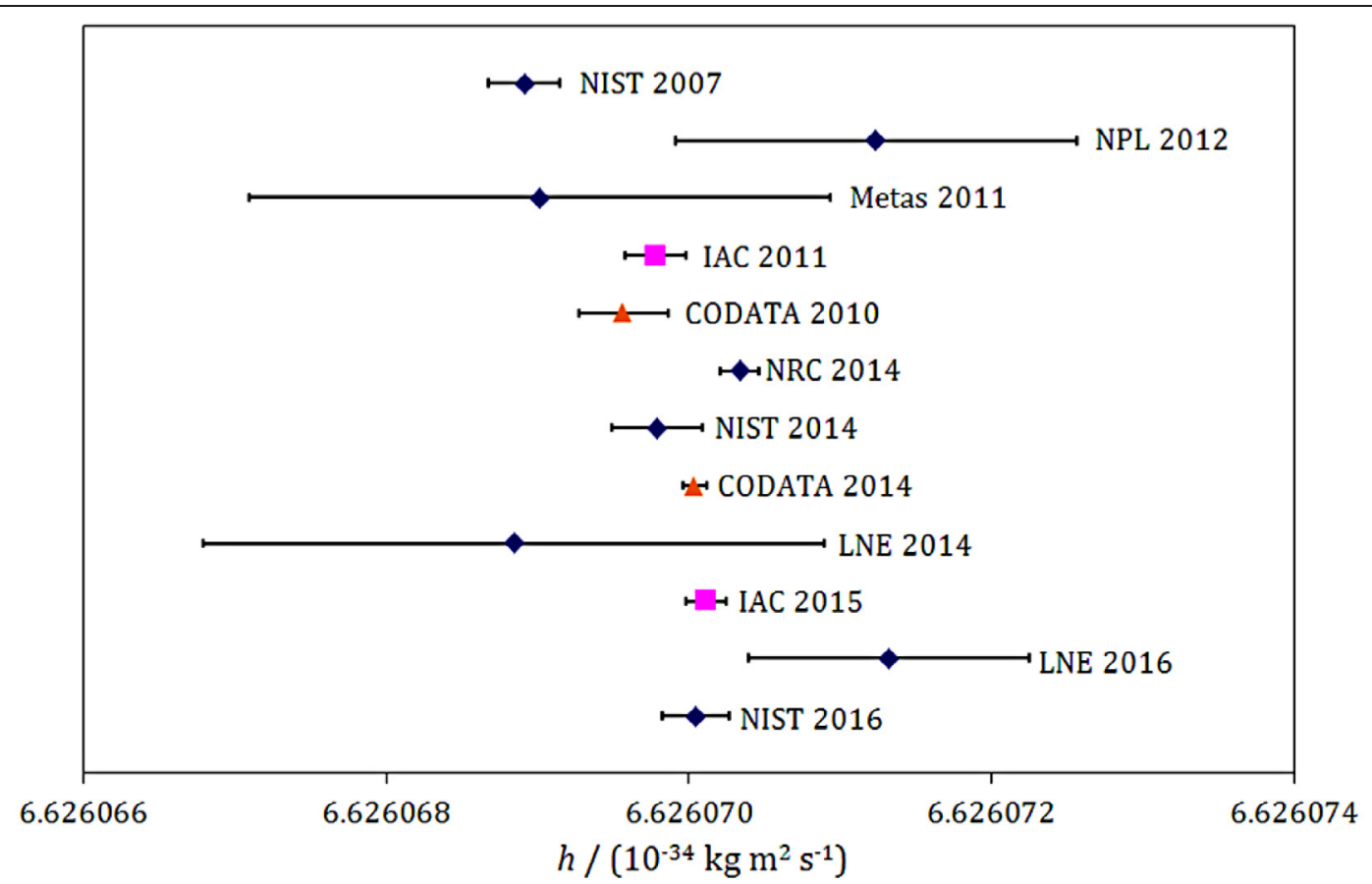

Fig. 3. - Représentation de la détermination de la constante de Planck $h$ par le LNE en 2014 et en 2016, accompagnée des dernières valeurs déterminées au moyen d'une balance du watt (carré sur pointe) ou d'une sphère de silicium-28 (carré), de même que les valeurs recommandées par le CODATA en 2010 et 2014 (triangle). Chaque valeur de $h$ est accompagnée de l'incertitude type standard associée. Les valeurs recommandées par le CODATA sont tributaires des résultats expérimentaux obtenus par différents laboratoires.

Ainsi la valeur CODATA 2014 est déduite des valeurs du NIST et du NRC et de leurs incertitudes. LNE (Laboratoire national de métrologie et d'essais - France) : 2014 [31], 2016 [32] ; NIST (National institute of standards and technology - USA) : 2007 [33],

2014 [34], 2016 [35] ; NPL (National Physical laboratory - UK) : 2012 [36]; Metas (Institut fédéral de métrologie - Suisse) :

2011 [37]; NRC (National research council - Canada) : 2014 [38] ; IAC (International Avogadro coordination) : 2011 [39] et $2015[40]$.

La chaîne en découlant, constituée de kilogrammes de travail en acier inoxydable ou en Udimet 720 permettra l'étalonnage de «poids» de $1 \mathrm{~kg}$ de classe $\mathrm{E} 1$ à $8 \times 10^{-8}$ pour les utilisateurs ainsi que la dissémination vers les multiples et sous-multiples du kilogramme.

Parmi les trois voies de dissémination de l'unité de masse, celle établie à partir d'étalons en Udimet 720 offre un avantage certain en réduisant les composantes d'incertitude sur la correction de poussée de l'air pour le transfert vers les étalons de travail et ceux des clients en acier inoxydable (car les masses volumiques de l'acier inoxydable et de l'Udimet 720 sont très voisines, respectivement $8000 \mathrm{~kg} \cdot \mathrm{m}^{-3}$ et $8140 \mathrm{~kg} \cdot \mathrm{m}^{-3}$ ). Des étalons en Udimet 720 couvrant le domaine de $1 \mathrm{~g}$ à $10 \mathrm{~kg}$ ont déjà été réalisés et permettront de garantir des incertitudes d'étalonnage compatibles avec les erreurs maximales tolérées des poids de classe E1. La voie utilisant un étalon en platine iridié pour la réalisation primaire avec la balance du watt devra être maintenue pendant une période transitoire de 10 ans pour vérifier la cohérence avec l'ancienne définition de l'unité de masse. Enfin, celle qui repose sur un étalon de référence en iridium, du fait de sa compatibilité magnétique avec la balance du watt, de sa masse volumique $\left(22500 \mathrm{~kg} \cdot \mathrm{m}^{-3}\right)$ proche de celle du platine iridié $\left(21500 \mathrm{~kg} \cdot \mathrm{m}^{-3}\right)$ et de sa grande dureté qui permet une utilisation plus intensive comparée au platine iridié, est une option indispensable vers l'amélioration de l'incertitude de la réalisation primaire de l'unité de masse.

Dans cette proposition de mise en pratique du kilogramme, la constitution d'un ensemble d'artefacts de $1 \mathrm{~kg}$ fait de différents matériaux serait un atout majeur pour examiner la cohérence et la stabilité de la chaîne de référence française construite à partir de la réalisation primaire. Cet ensemble permettra également de disposer d'une valeur de référence de masse à tout moment, y compris quand la balance du watt serait temporairement non opérationnelle.

De même, la participation à la première comparaison BIPM.M-K1, qui pourrait avoir lieu entre 2018 et 2020, sera essentielle pour le LNE. Au-delà de sa contribution à l'étalonnage de l'Ensemble of reference mass standards (ERMS) du BIPM, le LNE bénéficiera en retour d'une nouvelle valeur nationale, corrélée à la valeur des étalons primaires des autres participants, mais entachée d'une incertitude plus faible que celle associée à sa propre réalisation primaire. Ainsi, l'incertitude associée à l'étalonnage de $1 \mathrm{~kg}$ qui relève d'une des entrées des Calibration and Measurement Capabilities (CMC) du LNE publiées dans la banque de données du BIPM [49] resterait maitrisée à une valeur bien en deçà de l'incertitude exigée par l'OIML $\left(8 \times 10^{-8}\right)$. 


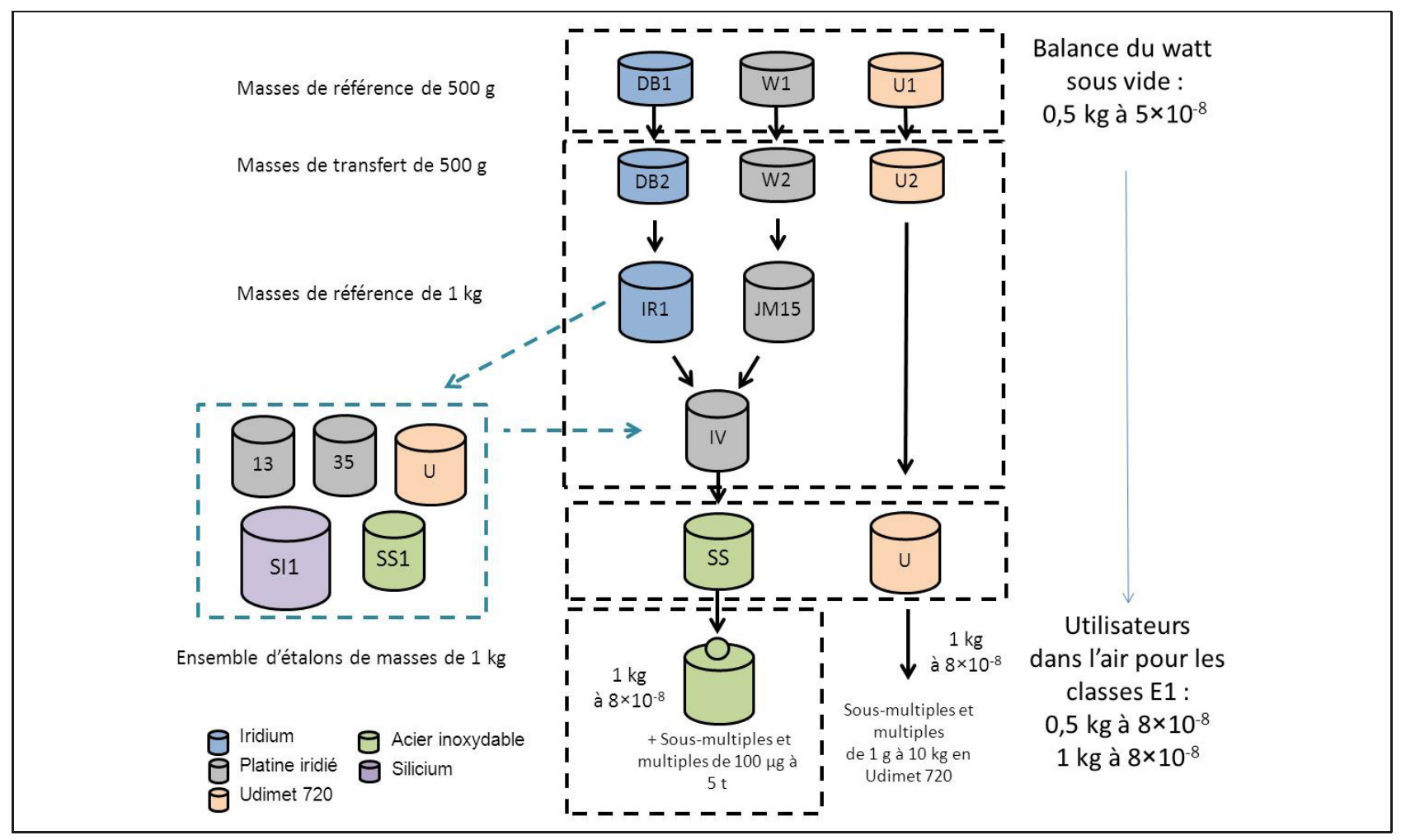

Fig. 4. - Possible système de dissémination de l'unité de masse assuré par la métrologie française après la redéfinition du kilogramme après 2018 .

\section{Conclusion}

Aujourd'hui, le LNE poursuit ses efforts pour améliorer les performances de sa balance du watt et prépare la transition vers la nouvelle chaîne de dissémination qui se fera parallèlement à la procédure de mise en pratique du kilogramme.

Atteindre une incertitude type relative inférieure à $5 \times 10^{-8}$ sur la réalisation du kilogramme à partir de la balance du watt placerait le LNE sur le plan mondial parmi les meilleurs laboratoires nationaux de métrologie détenteurs d'une référence primaire pour le kilogramme. Avec quelques autres membres de la Convention du mètre dotés d'un tel moyen primaire, la France pourrait alors participer à l'étalonnage des masses de l'ensemble des étalons de masse de référence du BIPM. La contribution du laboratoire à l'établissement de la valeur virtuelle de masse de l'ERMS du BIPM permettra en retour non seulement de vérifier le réglage de la balance du watt du LNE, mais surtout de réduire l'incertitude de dissémination et d'améliorer les CMC du LNE par rapport à une dissémination uniquement à partir de la balance du watt du LNE.

Le maintien de la balance du watt permettra par ailleurs de participer à des comparaisons bilatérales en Europe (METAS et/ou BIPM) ou internationale (NIST, NRC...), de piloter des comparaisons supplémentaires pour EURAMET, de proposer des raccordements de routine pour les pays n'ayant pas de réalisation primaire et de coopérer avec d'autres pays pour le développement de leur propre balance du watt.
Enfin, la balance du watt, contrairement aux étalons de masse à valeur ponctuelle, réalise non seulement l'unité de masse, mais également toute valeur de masse sur une étendue continue qui pourrait aller jusqu'à $10 \mathrm{~g}$. Elle pourra permettre de réaliser des valeurs de sousmultiples du kilogramme et ainsi disséminer directement ces sous-multiples. Cela offrira une grande simplification du raccordement des sous-multiples, une meilleure mâ̂trise de leur dérive et une amélioration significative des incertitudes d'étalonnage des sous-multiples.

\section{Références}

[1] BORDÉ C.J., «La réforme du système d'unités », La lettre de l'académie des sciences, 20, 2007, 21-27, christian.j. borde.free.fr/reforme.pdf (cf. pages 5-7).

[2] "The International Prototype", Site internet du BIPM : www.bipm.org/en/bipm/mass/image-ipk.html.

[3] «Comptes rendus de la troisième Conférence générale des poids et mesures (1901)». In : Conférence générale des poids et mesures (CGPM 3). Paris, 1901, site internet du BIPM : www.bipm.org/utils/common/pdf/CGPM/ CGPM3.pdf.

[4] «Comptes rendus de la première Conférence générale des poids et mesures (1889)». In : Conférence générale des poids et mesures (CGPM 1). Paris, 1890, site internet du BIPM : www.bipm.org/utils/common/pdf/CGPM/ CGPM1.pdf. 
[5] «Le Système international d'unités », 8 édition, 2006, brochure édité par le BIPM, mise à jour en 2014, accessible sur le site internet du BIPMURL : www.bipm.org/ en/publications/si-brochure/.

[6] GIRARD G., « Le nettoyage-lavage des prototypes du kilogramme au BIPM », 1990, accessible sur le site internet du BIPM : www.bipm.org/utils/fr/pdf/ Monographie1990-1-FR.pdf.

[7] MARTI K., Fuchs P. et RUSSI S., "Cleaning of mass standards: a comparison of new and old techniques", Metrologia, 49, 6, 2012, 628-634. DOI:10.1088/0026-1394/49/6/628.

[8] Silvestri Z., "Caractérisation physico-chimique de surface des étalons de masse », Thèse de doctorat de sciences du Conservatoire national des arts et métiers (CNAM), 31 oct. 2005, archives ouvertes TEL : tel-00011235.

[9] «Comptes rendus de la dixième Conférence générale des poids et mesures (1954) », Conférence générale des poids et mesures (CGPM 10), Paris, 1956, site internet du BIPM : www.bipm.org/utils/common/pdf/CGPM/ CGPM10.pdf.

[10] GIRARD G., "The third periodic verification of national prototypes of the kilogram (1988-1992)", Metrologia, 31, 4, 1994, 317, DOI: 10.1088/0026-1394/31/4/007.

[11] Milton M.J.T., DAVIS R. et FletcheR N., "Towards a new SI: a review of progress made since 2011”, Metrologia, 51, 3, 2014, R21, DOI:10.1088/0026-1394/51/3/R21.

[12] DAVIS R.S., "The stability of the SI unit of mass as determined from electrical measurements", Metrologia, 26, 1 , 1989, 75-76. DOI:10.1088/0026-1394/26/1/007.

[13] QUINN T., "The kilogram : the present state of our knowledge", IEEE Transactions on Instrumentation and Measurement, 40, 2, 199, 8185, DOI:10.1109/TIM.1990.1032888.

[14] GLASER M., "Tracing the atomic mass unit to the kilogram by ion accumulation", Metrologia, 40, 6, 2003, 376386, DOI:10.1088/0026-1394/40/6/009.

[15] KARSHENBoIM S.G., "On a natural definition of the kilogram and the ampere: the objectives and consequences", 2005, preprint arxiv:physics $/ 0507200$.

[16] MoHR P.J., "The fundamental constants and theory", Philosophical Transactions of the Royal Society A. Mathematical, Physical and Engineering Sciences, 363, 1834, 2005, 2123-2137, DOI:10.1098/rsta.2005.1641.

[17] Becker P., Bievre P.D., FuJiI K., Glaser M., Inglis B., LUEBbig H. et MANA G., "Considerations on future redefinitions of the kilogram, the mole and of other units", Metrologia, 44, 1, 2007, 1-14, DOI:10.1088/0026-1394/44/1/001.

[18] Mills I.M., MoHR P.J., QUINN T.J., TAYLOR B.N. et WILLIAMS E.R., "Redefinition of the kilogram, ampere, kelvin and mole: a proposed approach to implementing CIPM recommendation 1 (CI-2005)", Metrologia, 43, 3, 2006, 227-246, DOI:10.1088/0026-1394/43/3/006.
[19] Uzan J.P., « Dimensions et Constantes fondamentales », dans «Le Réel et ses dimensions », EDP Sciences, 2003.

[20] Davidson S., Berry J., AbBotT P., Marti K., GreEN R., MALENGO A. et NIELSE L.N., "Air-vacuum transfer; establishing traceability to the new kilogram", Metrologia, 53, 2016, A95-A113.

[21] Becker P., Bievre P.D., FuJII K., Glaser M., INGLis B., LUEBBiG H. et MANA G., "Considerations on future redefinitions of the kilogram, the mole and of other units", Metrologia, 44, 1, 2007, 1-14. DOI:10.1088/0026-1394/44/1/001.

[22] http://www.bipm.org/fr/measurement-units/new-si/\# communication.

[23] UZAN J.P., «Dimensions et Constantes fondamentales", in «Le Réel et ses dimensions », EDP Sciences, 2003.

[24] Mohr P.J., NewEll D.B. et TAYLOR B.N., "CODATA recommended values of the fundamental physical constants: 2014", Rev. Modern. Phys., 58, 2016.

[25] «Comptes rendus de la vingt-quatrième Conférence générale des poids et mesures (2011) », Conférence générale des poids et mesures (CGPM 24). Paris, 2013, http://www. bipm.org/utils/common/pdf/CGPM/CGPM24.pdf.

[26] «Comptes rendus de la vingt-cinquième Conférence générale des poids et mesures (2014) ». In : Conférence générale des poids et mesures (CGPM 24). Paris, 2015, http:// www.bipm.org/utils/common/pdf/CGPM/CGPM25.pdf.

[27] Eichenberger A., Geneves G. et Gournay P. "Determination of the Planck constant by means of a watt balance", Eur. Phys. J. Spec. Top., 172, 1, 2009, 363-383, DOI:10.1140/epjst/e2009-01061-3.

[28] Becker P., Bettin H., Danzebrink H.-U., Glaser M., Kuetgens U., Nicolaus A., Schiel D., BieVRe P.D., VALKIERS S. et TAYLOR P., "Determination of the Avogadro constant via the silicon route", Metrologia, 40, 5, 2003, page 271. DOI:10.1088/0026-1394/40/5/010.

[29] OIML, «Poids des classes $E_{1}, E_{2}, F_{1}, F_{2}, M_{1}, M_{1-2}$, $\mathrm{M}_{2}, \mathrm{M}_{2-3}$ et $\mathrm{M}_{3}$ - Partie 1 : Exigences métrologiques et techniques », 2004, http://www.oiml.org/fr/files/pdf_r/ r111-1-f04.pdf.

[30] KibBLE B.P., "Atomic Masses and Fundamental Constants", Proceedings of the Fifth International Conference on Atomic Masses and Fundamental Constants, Paris, juin 1975, Editors J.H. Sanders and A.H. Wapstra, New York : Plenum, 545-51, 1976.

[31] Thomas M., Espel P., Ziane D., Pinot P., Juncar P., Pereira Dos Santos F., Merlet S., PiQuemal F. et GENEVÈs G., 2015, "First determination of the Planck constant using the LNE watt balance", Metrologia, 52, 2015, 433-443.

[32] LNE first period report, CCM pilot study, mai 2016.

[33] SteineR R.L., Williams E.R., LiU R. et NEWEll D.B., "Uncertainty improvements of the NIST electronic kilogram”, IEEE Trans. Instr. Meas., 56, 2, 2007, 592-596, DOI:10.1109/TIM.2007.890590. 
[34] Schlamminger S., Steiner R.L., Haddad D., NeWEll D.B., SeIFERT F., CHAO L.S., LiU R., Williams E.R. et PRATT J.R., 2015. "A summary of the Planck constant measurements using a watt balance with a superconducting solenoid at NIST", Metrologia, 52, 2, L15, DOI:10.1088/0026-1394/52/2/L5.

[35] Haddad D., Seifert F., Chao L.S., Li S., Newell D.B., PratT J.R., Williams C. et SCHLAMminger S., "A precise instrument to determine the Planck constant, and the future kilogram", Rev. Sci. Instrum., 87, 2016, 061301, DOI:10.1063/1.4953825.

[36] Robinson I.A., "Towards the redefinition of the kilogram: a measurement of the Planck constant using the NPL Mark II watt balance", Metrologia, 49, 1, 2012, 113156, DOI:10.1088/0026-1394/49/1/016.

[37] EICHENBERge A.R, BAUMANN H., JEANNERET B., JeckelmanN B., Richard P. et Beer W., "Determination of the Planck constant measurements with the Metas watt balance with a superconducting solenoid at NIST", Metrologia, 48, 3, 2011, 133, DOI:10.1088/0026-1394/48/3/007.

[38] Sanchez C.A., Wood B.M., GReen R.G., Liard J.O. et INGLIS D., "A determination of Planck's using the NRC watt balance", Metrologia, 51, 2, 2014, S5, DOI:10.1088/0026-1394/51/2/S5.

[39] Andreas B., Azuma Y., Bartl G., Becker P., Bettin H., Borys M., Busch I., GRAY M., FUChS P., FuJII K., Fujimoto H., Kessler E., Krumrey M., Kuetgens U., Kuramoto N., Mana G., Manson P., Massa E., Mizushima S., Nicolaus A., PicARD A., PramanN A., RIENITZ O., SCHIEl D., VAlKIERS S. et WASEDA A., "Determination of the Avogadro Constant by Counting the Atoms in a 28Si Crystal", Phys. Rev. Lett., 106, 3, 2011, 030801, DOI:10.1103/PhysRevLett.106.030801.

Article reçu le 30 juin 2016, version révisée reçue le $1^{\text {er }}$ décembre 2016.
[40] Azuma Y., Barat P., Bartl G., BetTin H., Borys M., Busch I., CiBIK L., D’ AGOSTINo G., FUJII K., FUJimoto H., Hioki A., KrumRey M., KuETGENS U., KURAMOTO N., Mana G., Massa E., MeE R., Mizushima S., NARUKaWA T., Nicolaus A.,. PramanN A, RabB S.A., Rienitz O., Sasso C., Stock M., JR R.D.V., WASEDA A., WUNDRACK S. et ZAKEL S., "Improved measurement results for the Avogadro constant using a 28 Si-enriched crystal", Metrologia, 52, 2, 2015, 360, DOI:10.1088/0026-1394/52/2/360.

[41] http://iopscience.iop.org/00261394/focus/Focus_on_ Realization_Maintenance_and_Disseminationof_the_ Kilogram.

[42] Bettin H. et Schlamminger S., "Realization, maintenance and dissemination of the kilogram in the revised SI", 2016 Metrologia, 53, A1.

[43] Davis R., BaRat P. et Stock M., "A brief history of the unit of mass: continuity of successive definitions of the kilogram 2016", Metrologia, 53, A12.

[44] AвbotT P.J. et KUBARYCh Z.J., "The New Kilogram Definition and its Implications for High-Precision Mass Tolerance Classes", J. Res. Natl. Inst. Stand. Technol., 118, 2013, DOI:10.6028/jres.118.016.

[45] Glaser M., Borys M., Ratschko D. et Schwartz R., "Redefinition of the kilogram and the impact on its future dissemination", Metrologia, 47, 419, 2010, DOI:10.1088/0026-1394/47/4/007.

[46] http://www.npl.co.uk/content/ConPublication/6961.

[47] http://www.npl.co.uk/content/ConPublication/6960.

[48] http://www.newkilo.eu/reports/.

[49] Key Comparison Data Base (KCDB), http://kcdb.bipm. org/default_fr.asp.

[50] Los Arcos J.-M., Stock M., Wielgosz R., ARIAS F. et Milton M., "News from the BIPM laboratories: 2014", Metrologia, 52, 1, 2015, 155, DOI:10.1088/0026-1394/52/1/155. 